\title{
POWER GENERALIZED WEIBULL DISTRIBUTION BASED ON GENERALISED ORDER STATISTICS
}

\author{
Devendra Kumar ${ }^{1 *}$ and Neetu Jain ${ }^{2}$ \\ ${ }^{1}$ Department of Statistics, Central University of Haryana, India \\ ${ }^{2}$ Department of Statistics, University of Delhi, India
}

\begin{abstract}
The power generalized Weibull distribution due to Bagdonovacius and Nikulin (2002) is an alternative, and always provides better fits than the exponentiated Weibull family for modeling lifetime data. In this paper, we consider the generalized order statistics (GOS) from this distribution. We obtain exact explicit expressions as well as recurrence relations for the single, product and conditional moments of generalized order statistics from the power generalized Weibull distribution and then we use these results to compute the means and variances of order statistics and record values for samples of different sizes for various values of the shape and scale parameters.
\end{abstract}

Keywords: Power generalized Weibull distribution, generalized Order statistics, Recurrence relations, Single and product moments

\section{Introduction}

The two-parameter Weibull distribution is a very popular distribution that has been extensively used over the past decades for modeling data in reliability, engineering and biological studies. Because of its simplified applicability, it can take the form of either the exponential distribution, the Rayleigh distribution or can be skewed either positively or negatively. However, in cases where the hazard rates are bathtub or unimodal shapes, the Weibull distribution does not provide a reasonable parametric fit. To add more flexibility to Weibull distribution, many researchers developed many generalizations of the Weibull distribution by adding new parameters. However, with the increased number of parameters in the modified or extended version of the model, the forms of the survival and hazard functions have become more and more complicated and the estimation problems have become a challenging task [ see Bebbington et al. (2007), Mudholkar and Srivastava (1993), Ghitany et al. (2005), Wahed et al. (2009), Cordeiro et al. (2010), Silva et al. (2010), Risti'c and Balakrishnan (2012)]. The power generalized Weibull (PGW) distribution is another extension of the Weibull distribution which was first proposed by Bagdonova cius and Nikulin in (2002). Based on parameter values, the hazard function of PGW distribution can be constant, monotone (increasing and decreasing), bathtub shaped and upside down bathtub shaped. For more details about this extension, we refer the readers to Bagdonavi cius et al. 
(2006). Besides, it is a right skewed heavy tailed distribution which is not very common in life time model. The PGW family can be used as a possible alternative to the Exponentiated Weibull family for modeling lifetime data (Nikulin and Haghighi (2009)).

Bagdonovacius and Nikulin (2002) proposed the PGW distribution, which was further studied by Haghighi and Nikulin (2006), Alloyarova et al. (2007), Nikulin and Haghighi (2009) and Voinov et al. (2013). Haghighi and Nikulin (2004) proposed chi-squared type statistic to test the validity of the Power Generalized Weibull family based on Head-and-Neck cancer censored data. Alloyarova et al. (2007) constructed the Hsuan-Robson-Mirvaliev (HRM) statistic for testing the hypothesis based on moment-type estimators and investigated its properties. Nikulin and Haghighi (2009) ob- tained maximum likelihood estimates of the parameters and the flexibility of the model was shown by using Efron's (1988) head-and-neck cancer clinical trial data. Voinov et al. (2013) constructed modified chi-squared tests based on MLEs. Further, they studied power of the tests against ex- ponentiated Weibull, three-parameter Weibull, and generalized Weibull distributions using Monte Carlo simulations. Kumar and Sanku (2017) obtained the exact explicit expression and recurrence relations for generalized order statistics from power generalized Weibull distribution.

The recurrence relations and identities have great significance because they are useful in reduc- ing the number of operations necessary to obtain a general form for the function under consideration and they reduce the amount of direct computation, time and labour. This concept is well-established in the statistical literature, see Arnold et al. (1992) and Kumar (2015, 2017). Furthermore, they are used in characterizing distributions, which is an important area, permitting the identification of population distribution from the properties of the sample. $\operatorname{Kumar}(2013,2014,2015)$ have established recurrence relations for marginal and joint moment generating functions of lower generalized order statistics and generalized order statistics from Marshall-Olkin extended logistic, extended type II generalized logistic and type II exponentiated log-logistic distribution respectively. Bal- akrishnan et al. (2015) established some recurrence relations for single and product moments of order statistics of the complementary exponential-geometric distribution. Recently, Kumar and Sanku (2017)and Kumar et al. (2017) have established the relations for order statistics from ex- tended exponential and power generalized Weibull distribution respectively. Kumar and Sanku (2017) obtained the relations for single and product moments of generalized order statistics from extended exponential distribution. The computation of moments of order statistics is a challenging task for many distributions. For this reason, recursive computational methods are often sought.

A random variable $X$ has the power generalized Weibull (PGW) distribution with parameters $\alpha, \beta$ and $\lambda$, if its probability density function (pdf ) is

$$
\mathrm{f}(\mathrm{x})=\frac{\alpha}{\beta \lambda^{\alpha}} x^{\alpha-1}\left[1+\left(\frac{x}{\lambda}\right)^{\alpha}\right]^{\frac{1}{\beta}-1} e^{1-\left[1+\left(\frac{x}{\lambda}\right)^{\alpha}\right]^{\frac{1}{\beta}}} ; x>0, \alpha, \beta, \lambda>0
$$

the corresponding cumulative distribution function (cdf) is 


$$
\mathrm{F}(\mathrm{x})=1-e^{1-\left[1+\left(\frac{x}{\lambda}\right)^{\alpha}\right]^{\frac{1}{\beta}}} ; x>0, \alpha, \beta, \lambda>0
$$

The hazard function is given by

$$
\mathrm{h}(\mathrm{x})=\frac{f(x)}{1-F(x)}=\frac{\alpha}{\beta \lambda^{\alpha}} x^{\alpha-1}\left[1+\left(\frac{x}{\lambda}\right)^{\alpha}\right]^{\frac{1}{\beta}-1}, x>0
$$
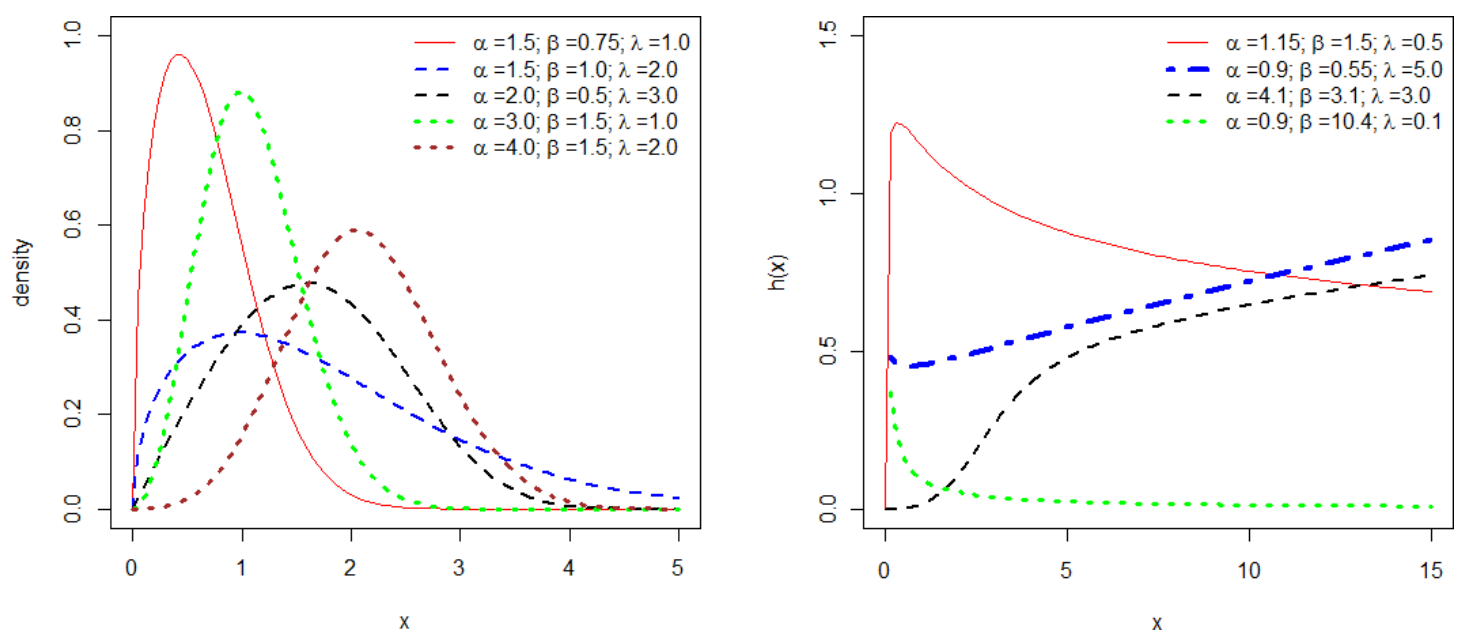

Figure 1: Probability density function and hazard function of PGW distribution for different values of $\alpha, \beta$ and $\lambda$.
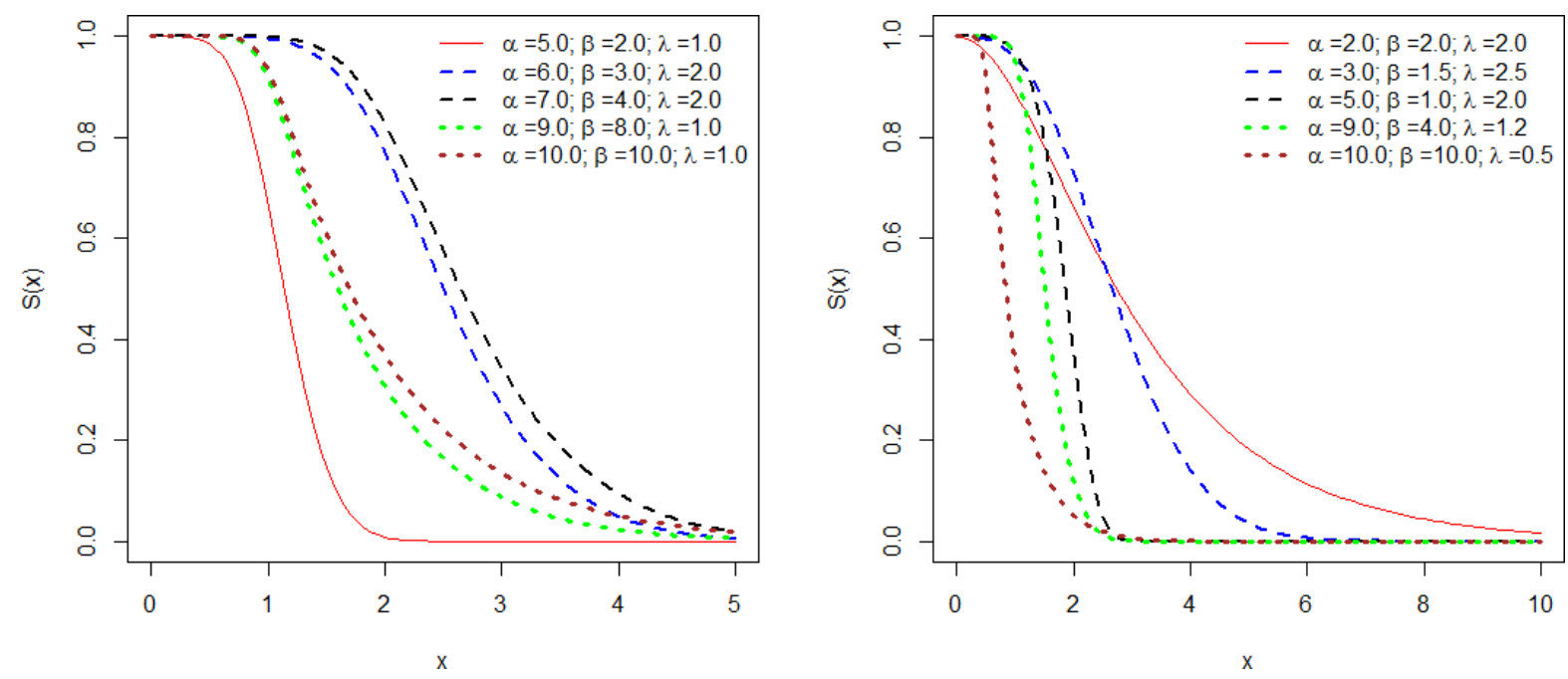

Figure 2: Survival function of PGW distribution for different values of $\alpha, \beta$ and $\lambda$.

If $\beta$ is real and non-integer and $|x|<1$, then (Gradshteyn and Ryzhik 2007, p. 25)

$$
(1+\mathrm{x})^{\beta}=\sum_{k=0}^{\infty}\left(\begin{array}{l}
\beta \\
k
\end{array}\right) x^{k} \text {. }
$$

One can observe from (1) and (2) that 


$$
\mathrm{f}(\mathrm{x})=\frac{\alpha}{\beta \lambda^{\alpha}} \sum_{u=0}^{\infty}\left(\begin{array}{c}
\frac{1}{\beta}-1 \\
u
\end{array}\right) \frac{x^{\alpha(u+1)-1}}{\lambda^{\alpha u}} \bar{F}(x),
$$

where $\bar{F}(x)=1-F(x)$. Now, the relation in (4) will be exploited to derive recurrence relations for the moments of generalized order statistics for the PGW distribution.

In our present paper, we define generalized order statistics. It will be shown that order statistics, record values and progressively Type II censored order statistics are special cases of generalized order statistics. First, we establish some explicit expressions for single moments, product moments and conditional moments of order statistics and record values. We also provide tabulations of means and variances of order statistics and record values for samples of different sizes of the shape and scale parameters.

The rest of the paper proceeds as follows: In Section 2, we describe briefly the preliminaries of generalized order statistics. In Section 3, we derive some explicit expressions and recurrence relations for single moments, product moments and conditional moments of generalized order statistics. In Section 4, we provide the characterization of PGW distribution based on recurrence relations of single moments of generalized order statistics. Tabulations of means and variances of order statistics and record values in Section 5. Finally, in Section 5, we make some concluding remarks.

\section{Generalized order statistics and preliminaries}

The concept of generalized order statistics was introduced by Kamps (1995). Since generalized order statistics is an unified approach of other ordered random scheme. Suppose $X(1, n, m, k), \ldots, X(n$, $\mathrm{n}, \mathrm{m}, \mathrm{k}),(\mathrm{k} \geq 1, \mathrm{~m}$ is a real number), are $\mathrm{n}$ GOS from an absolutely continuous cumulative distribution function $\operatorname{cdf} F(x)$ with probability density function $\operatorname{pdf} f(x)$, if their joint pdf is of the form

$$
\mathrm{k}\left(\prod_{j=1}^{n-1} \gamma_{j}\right)\left(\prod_{i=1}^{n-1}\left[1-F\left(x_{i}\right)\right]^{m} f\left(x_{i}\right)\right)\left[1-\mathrm{F}\left(x_{n}\right)\right]^{k-1} f\left(x_{n}\right)
$$

for $\mathrm{F}^{-1}(0)<x_{1}<x_{2}<\cdots<x_{n}<\mathrm{F}^{-1}(1)$. where $\gamma_{j}=\mathrm{k}+(\mathrm{n}-\mathrm{j})(\mathrm{m}+1)>0$ for $\mathrm{j}, 1 \leq \mathrm{j} \leq$ $\mathrm{n}, \mathrm{k}$ is a positive integer and $\mathrm{m} \geq-1$.If $\mathrm{m}=0$ and $\mathrm{k}=1$, we obtain the joint pdf of the order statistics. If $\mathrm{k}=1$ and $\mathrm{m}=-1$, we obtain the joint pdf of the first $\mathrm{n}$ record values of the identically and independently distributed (iid) random variables with cdf $\mathrm{F}(\mathrm{x})$ and corresponding pdf $\mathrm{f}(\mathrm{x})$. Other statistics contained as particular cases include sequential order statistics, progressively type II censored order statistics and Pfeifer's record values.

In view of (5), the marginal pdf of the $\mathrm{r}-$ th GOS, is given by

$$
f_{X(r, n, m, k)}(x)=\frac{C_{r-1}}{(r-1) !}[\bar{F}(x)]^{\gamma_{r-1}} f(x) g_{m}^{r-1}(F(x)) .
$$

The joint pdf of r-th and s-th GOS, is 


$$
\begin{aligned}
& f_{X(r, n, m, k), X(s, n, m, k)}(\mathrm{x}, \mathrm{y}) \\
& \quad=\frac{C_{s-1}}{(r-1) !(s-r-1) !}[\bar{F}(x)]^{m} f(x) g_{m}{ }^{r-1}(F(x)) \times\left[h_{m}(F(y))\right. \\
& \left.-h_{m}(F(x))\right]^{s-r-1}[\bar{F}(x)]^{\gamma_{s-1}} f(y),
\end{aligned}
$$

for $x<y$, where $\bar{F}(x)=1-F(x)$,

$$
\begin{gathered}
C_{r-1}=\prod_{i=1}^{r} \gamma_{i} \\
h_{m}(x)=\left\{\begin{array}{c}
-\frac{1}{m+1}(1-x)^{m+1}, m \neq-1 \\
-\ln (1-x), m=-1
\end{array}\right.
\end{gathered}
$$

and

$$
g_{m}(x)=h_{m}(x)-h_{m}(0), x \in[0,1)
$$

\section{Relations for single and product moments of generalized order statistics}

In this section, we derive explicit expressions and recurrence relations for single and product moments of generalized order statistics from the PGW distribution.

\subsection{Relations for single moments}

We shall first establish explicit expressions for jth single moments of the rth generalized order statistics, $\mathrm{E}\left(X_{r, n, m, k}{ }^{(j)}\right)=\mu_{r, n, m, k}{ }^{(j)}$. Theorem 1 gives an explicit expression for $1 \leq \mathrm{r} \leq \mathrm{n}$ and $\mathrm{j}=$ $0,1,2, \ldots$ Theorem 2 gives an explicit expression for $1 \leq \mathrm{r} \leq \mathrm{n}$ and $\mathrm{j}$ a negative integer.

Theorem1. For the PGW distribution given in (1) and for $1 \leq r \leq n$ and $j=0,1,2, \ldots$

$\mu_{r, n, m, k}^{(j)}=\frac{\lambda^{j} C_{r-1}}{(r-1) !(m+1)^{r-1}} \sum_{u=0}^{r-1} \sum_{l=0}^{\frac{j}{\alpha}}(-1)^{\mu+\frac{j}{\alpha}-l}\left(\begin{array}{c}r-1 \\ u\end{array}\right)\left(\begin{array}{c}\frac{j}{\alpha} \\ l\end{array}\right) \times \frac{e^{\gamma r-u}}{\gamma_{r-u}^{\beta l+1}} \Gamma\left(\beta l+1, \gamma_{r-u}\right)$,

and for $m=-1$

$$
\mu_{r, n,-1, k}^{(j)}=\frac{\lambda^{j} e^{k} k^{r}}{(r-1) !} \sum_{u=0}^{r-1} \sum_{l=0}^{j / \alpha} \frac{(-1)^{u+\frac{j}{\alpha}+r-l-1}}{k^{\beta l+u}}\left(\begin{array}{c}
r-1 \\
u
\end{array}\right)\left(\begin{array}{c}
j / \alpha \\
l
\end{array}\right) \times \Gamma(\beta l+u+1, k),
$$

where $\Gamma(\mathrm{a}, \mathrm{x})$ denotes the incomplete gamma function defined by $\Gamma(a, x)=\int_{x}^{\infty} t^{\alpha-1} e^{-t} d t$.

Proof. Using (6), we have

$$
\begin{gathered}
\mu_{r, n, m, k}^{(j)}=\frac{C_{r-1}}{(r-1) !} \int_{0}^{\infty} x^{j}[\bar{F}(x)]^{\gamma_{r}-1} g_{m}^{r-1}(F(x)) f(x) d x . \\
=\frac{C_{r-1}}{(r-1) !(m+1)^{r-1}} \sum_{u=0}^{r-1}(-1)^{u}\left(\begin{array}{c}
r-1 \\
u
\end{array}\right) \int_{0}^{\infty} x^{j}[1-F(x)]^{\gamma_{r-u}-1} f(x) d x
\end{gathered}
$$




$$
\begin{gathered}
=\frac{\alpha}{\beta \lambda^{\alpha}} \frac{C_{r-1}}{(r-1) !(m+1)^{r-1}} \sum_{u=0}^{r-1}(-1)^{u}\left(\begin{array}{c}
r-1 \\
u
\end{array}\right) e^{\gamma_{r}-u} \times \int_{0}^{\infty} x^{j+\alpha-1}\left[1+\left(\frac{x}{\lambda}\right)^{\alpha}\right]^{\frac{1}{\beta}-1} \exp \left[-\gamma_{r-u}\{1\right. \\
\left.\left.+\left(\frac{x}{\lambda}\right)^{\alpha}\right\}^{\frac{1}{\beta}}\right] \mathrm{dx} \\
=\frac{C_{r-1} \lambda^{j}}{(r-1) !(m+1)^{r-1}} \sum_{u=0}^{r-1} \sum_{l=0}^{\frac{j}{\alpha}}(-1)^{u+\frac{j}{\alpha}-l}\left(\begin{array}{c}
r-1 \\
u
\end{array}\right)\left(\begin{array}{c}
\frac{j}{\alpha} \\
l
\end{array}\right) \frac{e_{r-u}^{\gamma}}{\gamma_{r-u}^{\beta l+1}} \times \int_{\gamma_{r-u}}^{\infty} y^{\beta l} e^{-y} d y,
\end{gathered}
$$

where $y=\left(\gamma_{r-u}\right)\left[1+\left(\frac{x}{\lambda}\right)^{\alpha}\right]^{\frac{1}{\beta}}$. The result follows from the definition of the incomplete gamma function. For $m=-1$

$$
\left.\mu_{r, n,-1, k}^{(j)}=\frac{\lambda^{j} e^{k} k^{r}}{(r-1) !} \sum_{u=0}^{r-1} \sum_{l=0}^{j / \alpha}-1\right)^{u+\frac{j}{\alpha}+r-l-1}\left(\begin{array}{c}
r-1 \\
u
\end{array}\right)\left(\begin{array}{c}
j / \alpha \\
l
\end{array}\right) \times k^{-\beta l-u} \int_{k}^{\infty} y^{\beta l+u} e^{-y} d y,
$$

where $\mathrm{y}=\mathrm{k}\left[1+\left(\frac{x}{\lambda}\right)^{\alpha}\right]^{\frac{1}{\beta}}$. The result follows from the definition of the incomplete gamma function.

In particular, the mean and the variance of generalised order statistic are

$$
\begin{gathered}
\mu_{r, n, m, k}=\mu_{r, n, m, k}^{(l)} \\
=\lambda \frac{C_{r-1}}{(r-1) !(m+1)^{r-1}} \sum_{u=0}^{r-1} \sum_{l=0}^{\frac{1}{\alpha}}(-1)^{u+\frac{1}{\alpha}-l}\left(\begin{array}{c}
r-1 \\
u
\end{array}\right)\left(\begin{array}{c}
\frac{1}{\alpha} \\
l
\end{array}\right) \times \frac{e^{\gamma r-u}}{\gamma_{r-u}^{\beta l+1}} \Gamma\left(\beta l+1, \gamma_{r-u}\right),
\end{gathered}
$$

and

$$
\begin{gathered}
\sigma_{r, n, m, k}^{2}=\mu_{r, n, m, k}^{(2)}-\left(\mu_{r, n, m, k}\right)^{2} \\
=\lambda^{2} \frac{C_{r-1}}{(r-1) !(m+1)^{r-1}} \sum_{u=0}^{r-1} \sum_{l=0}^{\frac{2}{\alpha}}(-1)^{u+\frac{2}{\alpha}-l}\left(\begin{array}{c}
r-1 \\
u
\end{array}\right)\left(\begin{array}{c}
\frac{2}{\alpha} \\
k
\end{array}\right) \\
\times \frac{e^{\gamma_{r-u}}}{\gamma_{r-u}^{\beta l+1}} \Gamma\left(\beta l+1, \gamma_{e-u}\right)-\left(\mu_{r, n, m, k}\right)^{2},
\end{gathered}
$$

respectively.

\section{Special cases}

1) If we put $m=0, k=1$ in (8), we get explicit expressions for ordinary order statistics $\left(\gamma_{r}=n-r+\right.$ 1), we have

$$
\mu_{r: n}^{(j)}=C_{r: n} \lambda^{j} \sum_{u=0}^{r-1} \sum_{l=0}^{j / \alpha}(-1)^{u+\frac{j}{\alpha}-l}\left(\begin{array}{c}
r-1 \\
u
\end{array}\right)\left(\begin{array}{c}
j / \alpha \\
l
\end{array}\right) \times \frac{e^{n-r+u+1}}{(n-r+u+1)^{\beta l+1}} \Gamma(\beta l+1, n-r+u+1)
$$

which is the result obtained by by Kumar and Sanku (2017).

In particular, the mean order statistics and the variance order statistics are 


$$
\begin{gathered}
\mu_{r: n}^{(j)}=C_{r: n} \lambda \sum_{u=0}^{r-1}\left(\begin{array}{c}
r-1 \\
u
\end{array}\right) e^{n-r+u+1}\left\{\frac{(-1)^{u+\frac{1}{\alpha}}}{n-r+u+1} \Gamma(1, n-r+u+1)\right. \\
\left.+\frac{(-1)^{u}}{(n-r+u+1)^{1+\frac{\beta}{\alpha}}} \Gamma\left(\frac{\beta}{\alpha}+1, n-r+u+1\right)\right\}
\end{gathered}
$$


and

$$
\begin{gathered}
\sigma_{r: n}^{2}=\mu_{r: n}^{(2)}-\left[\mu_{r: n}^{(1)}\right]^{2} \\
=C_{r: n} \lambda^{2} \sum_{u=0}^{r-1}\left(\begin{array}{c}
r-1 \\
u
\end{array}\right) e^{n-r+u-1}\left\{\frac{(-1)^{u+\frac{2}{\alpha}}}{n-r+u+1} \Gamma(1, n-r+u+1)\right. \\
+\frac{(-1)^{u+\frac{1}{\alpha}}}{(n-r+u+1)^{1+\frac{\beta}{\alpha}}} \Gamma\left(\frac{\beta}{\alpha}+1, n-r+u+1\right) \\
\left.+\frac{(-1)^{u}}{(n-r+u+1)^{1+\frac{2 \beta}{\alpha}}} \Gamma\left(\frac{2 \beta}{\alpha}+1, n-r+u+1\right)\right\}-\left(\mu_{r: n}^{(l)}\right)^{2},
\end{gathered}
$$

respectively.

3) If we put $\mathrm{k}=1$ in (9), ordinary record values we have

$$
\mu_{U(r)}^{(j)}=\frac{e \lambda^{j}}{(r-1) !} \sum_{u=0}^{r-1} \sum_{l=0}^{\frac{j}{\alpha}}(-1)^{u+\frac{j}{\alpha}-1+r-l}\left(\begin{array}{c}
r-1 \\
u
\end{array}\right)\left(\begin{array}{c}
\frac{j}{\alpha} \\
l
\end{array}\right) \Gamma(\beta l+u+1,1) .
$$

In particular, the mean record statistics and the variance record statistics are

$$
\mu_{U(r)}^{(1)}=\frac{e \lambda}{(r-1) !} \sum_{u=0}^{r-1}\left(\begin{array}{c}
r-1 \\
u
\end{array}\right)\left\{(-1)^{u+\frac{1}{\alpha}-1+r} \Gamma(u+1,1)+(-1)^{u-1+r} \Gamma\left(\frac{\beta}{\alpha}+u+1,1\right)\right\}
$$

and

$$
\begin{aligned}
\sigma_{U_{(r)}}^{2}=\mu_{U_{(r)}}^{(2)}- & {\left[\mu_{U_{(r)}}^{(1)}\right]^{2} } \\
& =\frac{e \lambda}{(r-1) !} \sum_{u=0}^{r-1}\left(\begin{array}{c}
r-1 \\
u
\end{array}\right)\left\{(-1)^{u+\frac{2}{\alpha}-1+r} \Gamma(u+1,1)\right. \\
& \left.+(-1)^{u+\frac{1}{\alpha}-1+r}\left(\begin{array}{c}
\frac{2}{\alpha} \\
\frac{l}{\alpha}
\end{array}\right) \Gamma\left(\frac{\beta}{\alpha}+u+1,1\right)+(-1)^{u-1+r} \Gamma\left(\frac{2 \beta}{\alpha}+u+1,1\right)\right\} \\
& -\left[\mu_{U_{(r)}}^{(1)}\right]^{2},
\end{aligned}
$$

respectively. 
Theorem 2. For the PGW distribution given in (1) and for $1 \leq r \leq n$ and $j$ a negative integer,

$$
\mu_{r, n, m, k}^{(j)}=\frac{\lambda^{j} C_{r-1}}{(r-1) !(m+1)^{r-1}} \sum_{u=0}^{r-1} \sum_{l=0}^{\infty}(-1)^{u+\frac{j}{\alpha}-l}\left(\begin{array}{c}
r-1 \\
u
\end{array}\right)\left(\begin{array}{c}
j / \alpha \\
l
\end{array}\right) \times \frac{e^{\gamma_{r-u}}}{\gamma_{r-u}^{\beta l+1}} \Gamma\left(\beta l+1, \gamma_{r-u}\right),
$$

and for $m=-1$

$$
\mu_{r, n,-1, k}^{(j)}=\frac{\lambda^{j} e^{k} k^{r}}{(r-1) !} \sum_{u=0}^{r-1} \sum_{l=0}^{\infty} \frac{(-1)^{u+\frac{j}{\alpha}+r-l-1}}{k^{\beta l+u}}\left(\begin{array}{c}
r-1 \\
u
\end{array}\right)\left(\begin{array}{c}
j / \alpha \\
l
\end{array}\right) \times \Gamma(\beta l+u+1, k) .
$$

Proof. Similar to the proof of Theorem1.

Theorem 3 establishes a recurrence relation for $\mu_{r, n, m, k}^{(j)}$. This result holds for positive as well as negative $\mathrm{j}$.

Theorem3. For the PGW distribution given in (1) and for $1 \leq r \leq n$,

$$
\mu_{r, n, m, k}^{(j)}=\frac{\alpha}{\beta} \sum_{u=0}^{\infty}\left(\begin{array}{c}
\frac{1}{\beta}-1 \\
u
\end{array}\right) \frac{\lambda^{-\alpha(u+1)}}{j+\alpha(u+1)} \times\left[\gamma_{r} \mu_{r, n, m, k}^{(j+\alpha(u+1))}-(r-1) \mu_{r-1, n, m, k}^{(j+\alpha(u+1))}\right], \beta>1
$$

Throughout, we follow the conventions that $\mu_{0, n, m, k}^{(j)}=0$ for $\mathrm{n} \geq 1$ and $\mu_{0, n, m, k}^{(j)}=1$ for $1 \leq \mathrm{r}<\mathrm{n}$.

Proof. For $1 \leq \mathrm{r} \leq \mathrm{n}$. we have from (4) and (5)

$$
\begin{gathered}
\mu_{r, n, m, k}^{(j)}=\frac{C_{r-1}}{(r-1) !} \int_{0}^{\infty} x^{j}[\bar{F}(x)]^{\gamma_{r-1}} g_{m}^{r-1}(F(x)) f(x) d x \\
=\frac{\alpha}{\beta \lambda^{\alpha}} \frac{C_{r-1}}{(r-1) !} \sum_{u=0}^{\infty}\left(\begin{array}{c}
\frac{1}{\beta}-1 \\
\mu
\end{array}\right) \frac{1}{\lambda^{\alpha u}} \int_{0}^{\infty} x^{j+\alpha(u+1)-1}[\bar{F}(x)]^{\gamma_{r}} g_{m}^{r-1}(F(x)) d x .
\end{gathered}
$$

Now integrating by parts, we obtain

$$
\begin{aligned}
\mu_{r, n, m, k}^{(j)}=\frac{\alpha}{\beta \lambda^{\alpha}} & \frac{C_{r-1}}{(r-1) !} \sum_{0}^{\infty}\left(\begin{array}{c}
\frac{1}{\beta}-1 \\
u
\end{array}\right) \frac{1}{\lambda^{\alpha u}} \\
& \times\left\{\frac{\gamma_{r}}{j+\alpha(u+1)} \int_{0}^{\infty} x^{j+\alpha(u+1)-1}[\bar{F}(x)]^{\gamma_{r}-1} g_{m}^{r-1}(F(x)) f(x) d x\right. \\
& \left.-\frac{r-1}{j+\alpha(u+1)} \int_{0}^{\infty} x^{j+\alpha(u+1)}[\bar{F}(x)]^{\gamma_{r}-m} g_{m}^{r-2}(F(x)) f(x) d x\right\} .
\end{aligned}
$$

The result follows.

In particular, upon setting $\mathrm{r}=1$ in Theorem 3 , we deduce the following result.

Corollary 1. For PGW distribution,

$$
\mu_{1, n, m, k}^{(j)}=\frac{\alpha}{\beta} \gamma_{1} \sum_{u=0}^{\infty}\left(\begin{array}{c}
\frac{1}{\beta}-1 \\
u
\end{array}\right) \frac{\lambda^{-\alpha(u+1)}}{j+\alpha(u+1)} \mu_{1, n, m, k}^{(j+\alpha(u+1))}
$$




\section{Special Cases.}

1) For $m=0, k=1$ in (17) and (18), we get the recurrence relations for ordinary order statistics

$$
\mu_{r: n}^{(j)}=\frac{\alpha}{\beta} \sum_{u=0}^{\infty}\left(\begin{array}{c}
\frac{1}{\beta}-1 \\
u
\end{array}\right) \frac{\lambda^{-\alpha(u+1)}}{j+\alpha(u+1)} \times\left[(n-r+1) \mu_{r: n}^{(j+\alpha(u+1))}-(r-1) \mu_{r-1: n}^{(j+\alpha(u+1))}\right],
$$

and

$$
\mu_{1: n}^{(j)}=\frac{\alpha}{\beta} n \sum_{u=0}^{\infty}\left(\begin{array}{c}
\frac{1}{\beta}-1 \\
u
\end{array}\right) \frac{\lambda^{-\alpha(u+1)}}{j+\alpha(u+1)} \mu_{1: n}^{(j+\alpha(u+1))}
$$

2) For $m=-1, k \geq 1$ in (17) and (18), we get recurrence relations for record values

$$
\mu_{U_{(r): k}}^{(j)}=\frac{\alpha}{\beta} \sum_{u=0}^{\infty}\left(\begin{array}{c}
\frac{1}{\beta}-1 \\
u
\end{array}\right) \frac{\lambda^{-\alpha(u+1)}}{j+\alpha(u+1)}\left[k \mu_{U_{(r): k}}^{(j+\alpha(u+1))}-(r+1) \mu_{U(r-1): k}^{(j+\alpha(u+1))}\right],
$$

and

$$
\mu_{U(1): k}^{(j)}=k \frac{\alpha}{\beta} \sum_{u=0}^{\infty}\left(\begin{array}{c}
\frac{1}{\beta}-1 \\
u
\end{array}\right) \frac{\lambda^{-\alpha(u+1)}}{j+\alpha(u+1)} \mu_{U_{(1): k}}^{(j+\alpha(u+1))}
$$

\subsection{Relations for product moments}

We shall first establish explicit expressions for the product moment of ith and jth generalized order statistics, $\mathrm{E}\left[X_{r, s, n, m, k}^{(i, j)}\right]=\mu_{r, s, n, m, k}^{(i, j)}$.Theorem 4 gives an explicit expression for $1 \leq r<s \leq n$ and $i, j=0,1,2, \ldots$ Theorem 5 gives an explicit expression for $1 \leq r<s \leq n, i=0,1,2, \ldots$ and $\mathrm{j}$ a negative integer. Theorem 6 gives an explicit expression for $1 \leq r<s \leq n, j=0,1,2, \ldots$ and $i$ a negative integer. Theorem 7 gives an explicit expression for $1 \leq r<s \leq n$ and both $i$ and $j$ negative integers.

Theorem 4. For the PGW distribution given in (1) and for $1 \leq r<s \leq n, i, j=0,1,2, \ldots$,

$$
\begin{aligned}
\mu_{r, s ; n, m, k}^{(i, j)}=\frac{\lambda^{i+j} C_{s-1}}{(r-1) !(s-r-1) !(m+1)^{s-2}} \sum_{u=0}^{r-1} \sum_{v=0}^{s-r-1} \sum_{p=0}^{j / \alpha} \sum_{q=0}^{i / \alpha}(-1)^{u+v+\frac{(i+j)}{\alpha}-p-q} \\
\quad \times\left(\begin{array}{c}
r-1 \\
u
\end{array}\right)\left(\begin{array}{c}
s-r-1 \\
v
\end{array}\right)\left(\begin{array}{c}
j / \alpha \\
p
\end{array}\right)\left(\begin{array}{c}
i / \alpha \\
q
\end{array}\right) \frac{e^{\gamma_{r-u}}}{(\beta q+1) \gamma_{r-u}^{2+\beta(p+q)}} \\
\quad \times 2 F_{1}\left(1,2+\beta(p+q) ; 2+\beta q ; \frac{(u+s-r-v)(m+1)}{\gamma_{r-u}}\right), m \neq-1
\end{aligned}
$$


and for $\mathrm{m}=-1$

$$
\begin{aligned}
\mu_{r, s ; n,-1, k}^{(i, j)}=\frac{\lambda^{i+j} e^{k} k^{s}}{(r-1) !(s-r-1) !} \sum_{u=0}^{r-1} \sum_{v=0}^{s-r-1} \sum_{p=0}^{j / \alpha} \sum_{q=0}^{i / \alpha}(-1)^{\frac{i+j}{\alpha}+r+v-u-p-q-1} \\
\quad \times\left(\begin{array}{c}
r-1 \\
u
\end{array}\right)\left(\begin{array}{c}
s-r-1 \\
v
\end{array}\right)\left(\begin{array}{c}
j / \alpha \\
p
\end{array}\right)\left(\begin{array}{c}
i / \alpha \\
q
\end{array}\right) \frac{\Gamma(\beta(p+q)+s-r+u+1)}{(\beta q+u+v+1)} \\
\times 2 F_{1}(1, \beta(p+q)+s-r+u+1 ; \beta q+u+v+2 ; 1),
\end{aligned}
$$

where $2 F_{1}(a, b ; c ; x)$ denotes the Gauss hypergeometric function defined by

where $(e)_{k}=e(e+1) \ldots(e+k-1)$

$$
2 F_{1}(a, b ; c ; x)=\sum_{k=0}^{\infty} \frac{(a)_{k}(b)_{k}}{(c)_{k}} \frac{x^{k}}{k !},
$$

Proof. From (7), we have

$$
\begin{aligned}
& \mu_{r, s ; n, m, k}^{(i, j)} \\
& =\frac{C_{s-1}}{(r-1) !(s-r-1) !} \int_{0}^{\infty} \int_{x}^{\infty} x^{i} y^{j}[\bar{F}(\mathrm{x})]^{m} f(x) g_{m}^{r-1}(F(x)) \times\left[g_{m}(F(y))\right. \\
& \left.-g_{m}(F(x))\right]^{s-r-1}[\bar{F}(\mathrm{y})]^{\gamma_{s}-1} f(y) d y d x \\
& =\frac{C_{s-1}}{(r-1) !(s-r-1) !(m+1)^{s-2}} \sum_{u=0}^{r-1} \sum_{v=0}^{s-r-1}(-1)^{u+v}\left(\begin{array}{c}
r-1 \\
u
\end{array}\right)\left(\begin{array}{c}
s-r-1 \\
v
\end{array}\right) \\
& \times \int_{0}^{\infty} \int_{x}^{\infty} x^{i} y^{j}[\bar{F}(\mathrm{x})]^{(s-r+u-v)(m+1)-1}[\bar{F}(\mathrm{y})]^{\gamma_{s-v}-1} f(x) f(y) d y d x \\
& =\frac{\alpha^{2} C_{s-1}}{(r-1) !(s-r-1) !(m+1)^{s-2} \beta^{2} \lambda^{2 \alpha}} \sum_{u=0}^{r-1} \sum_{v=0}^{s-r-1}(-1)^{u+v}\left(\begin{array}{c}
r-1 \\
u
\end{array}\right)\left(\begin{array}{c}
s-r-1 \\
v
\end{array}\right) \\
& \times \int_{0}^{\infty} \int_{x}^{\infty} x^{i+\alpha-1} y^{j+\alpha-1}\left[1+\left(\frac{x}{\lambda}\right)^{\alpha}\right]^{\frac{1}{\beta}-1}\left[1+\left(\frac{y}{\lambda}\right)^{\alpha}\right]^{\frac{1}{\beta}-1} \times \exp (\{1-[1 \\
& \left.\left.\left.+\left(\frac{x}{\lambda}\right)^{\alpha}\right]^{\frac{1}{\beta}-1}\right\}(s-r+u-v)(m+1)\right) \times \exp \left(\left\{1-\left[1+\left(\frac{y}{\lambda}\right)^{\alpha}\right]^{\frac{1}{\beta}-1}\right\} \gamma_{s-v}\right) d y d x \\
& \left.\left.+\left(\frac{x}{\lambda}\right)^{\alpha}\right]^{\frac{1}{\beta}}(s-r+u-v)(\mathrm{m}+1)\right) \times \Gamma\left(\beta \mathrm{p}+1,\left[1+\left(\frac{x}{\lambda}\right)^{\alpha}\right]^{\frac{1}{\beta}} \gamma_{s-v}\right) d x \\
& \times\left(\begin{array}{c}
r-1 \\
u
\end{array}\right)\left(\begin{array}{c}
s-r-1 \\
v
\end{array}\right)\left(\begin{array}{c}
\frac{j}{\alpha} \\
p
\end{array}\right) e^{\gamma_{r-u}{ }^{\beta p+1}} \times \int_{x-1}^{\infty} x^{i+\alpha-1}[1 \\
& =
\end{aligned}
$$




$$
\begin{aligned}
=\frac{\lambda^{i+j} C_{s-1}}{(r-1) !(s-} & r-1) !(m+1)^{\beta q+s-1} \\
\sum_{u=0}^{r-1} & \sum_{v=0}^{s-r-1} \sum_{p=0}^{\frac{j}{\alpha}} \sum_{q=0}^{\frac{i}{\alpha}}(-1)^{u+v+\frac{i+j}{\alpha}-p-q} \\
& \times\left(\begin{array}{c}
r-1 \\
u
\end{array}\right)\left(\begin{array}{c}
s-r-1 \\
v
\end{array}\right)\left(\begin{array}{c}
\frac{j}{\alpha} \\
p
\end{array}\right)\left(\begin{array}{c}
\frac{i}{\alpha} \\
q
\end{array}\right) \frac{e^{\gamma_{r-u}}}{\gamma_{s-v}^{\beta p+1}(s-r+u-v)^{\beta q+1}} \\
& \times \int_{(s-r+u-v)(m+1)}^{\infty} w^{\beta q} e^{-w} \Gamma\left(\beta q+1, \frac{\omega \gamma_{r-v}}{(s-r+u-v)(m+1)}\right) d w
\end{aligned}
$$

where $\mathrm{z}=\gamma_{s-v}\left[1+\left(\frac{y}{\lambda}\right)^{\alpha}\right]^{\frac{1}{\beta}}$ and $\mathrm{w}=(\mathrm{u}+\mathrm{s}-\mathrm{r}-\mathrm{v})(\mathrm{m}+1)\left[1+\left(\frac{x}{\lambda}\right)^{\alpha}\right]^{\frac{1}{\beta}}$. The result follows by using equation (6.455.1) in Gradshteyn and Ryzhik (2007) to calculate the integral in (25).

For $\mathrm{m}=-1$

$$
\begin{aligned}
& \mu_{r, s ; n, m, k}^{(i, j)}=\frac{k^{s}}{(r-1) !(s-r-1) !} \int_{0}^{\infty} \int_{x}^{\infty} x^{i} y^{j}[-\ln \bar{F}(x)]^{r-1} \frac{f(x)}{\bar{F}(x)} \times[-\ln \bar{F}(y)+ \\
& \ln \bar{F}(x)]^{s-r-1}[\bar{F}(y)]^{k-1} f(y) d y d x= \\
& \frac{\alpha^{2} k^{s} e^{k}}{(r-1) !(s-r-1) ! \beta^{2} \lambda^{2 \alpha}} \sum_{u=0}^{r-1} \sum_{v=0}^{s-r-1}(-1)^{r-1-u+v}\left(\begin{array}{c}
r-1 \\
u
\end{array}\right)\left(\begin{array}{c}
s-r-1 \\
v
\end{array}\right) \times \int_{0}^{\infty} \int_{x}^{\infty} x^{i+\alpha-1} y^{j+\alpha-1}[1+ \\
& \left.\left.\left(\frac{x}{\lambda}\right)^{\alpha}\right]^{\frac{u+v}{\beta}+\frac{1}{\beta}-1} \times\left[1+\left(\frac{y}{\lambda}\right)^{\alpha}\right]^{\frac{s-r-1-v}{\beta}+\frac{1}{\beta}-1} \exp \left(-\mathrm{k}\left\{\left[1+\left(\frac{y}{\lambda}\right)^{\alpha}\right)\right]^{\frac{1}{\beta}}\right\}\right) \mathrm{dy} d x= \\
& \frac{\alpha k^{s} e^{k} \lambda^{j}}{(r-1) !(s-r-1) ! \beta \lambda^{\alpha}} \sum_{u=0}^{r-1} \sum_{v=0}^{s-r-1} \sum_{p=0}^{\frac{j}{\alpha}} \frac{(-1)^{r-1-u+v+\frac{j}{\alpha}-p}}{k^{\beta p+s-r-v}} \times\left(\begin{array}{c}
r-1 \\
u
\end{array}\right)\left(\begin{array}{c}
s-r-1 \\
v
\end{array}\right)\left(\begin{array}{c}
\frac{j}{\alpha} \\
p
\end{array}\right) \times \\
& \int_{0}^{\infty} x^{i+\alpha-1}\left[1+\left(\frac{x}{\lambda}\right)^{\alpha}\right]^{\frac{u+v}{\beta}+\frac{1}{\beta}-1} \int_{k\left[1+\left(\frac{x}{\lambda}\right)^{\alpha}\right]^{\frac{1}{\beta}} z^{\beta p+s-r-1-v} e^{-z} d z d x=}^{\infty} d x \\
& \frac{\alpha k^{s} e^{k} \lambda^{j}}{(r-1) !(s-r-1) ! \beta \lambda^{\alpha}} \sum_{u=0}^{r-1} \sum_{v=0}^{s-r-1} \sum_{p=0}^{\frac{j}{\alpha}} \frac{(-1)^{r-1-u+v+\frac{j}{\alpha}-p}}{k^{\beta p+s-r-v}} \times\left(\begin{array}{c}
r-1 \\
u
\end{array}\right)\left(\begin{array}{c}
s-r-1 \\
v
\end{array}\right)\left(\begin{array}{c}
\frac{j}{\alpha} \\
p
\end{array}\right) \times \\
& \int_{0}^{\infty} x^{i+\alpha-1}\left[1+\left(\frac{x}{\lambda}\right)^{\alpha}\right]^{\frac{u+v}{\beta}+\frac{1}{\beta}-1} \Gamma\left(\beta p+s-r-v, k\left[1+\left(\frac{x}{\lambda}\right)^{\alpha}\right]^{\frac{1}{\beta}}\right) d x= \\
& \frac{k^{s} e^{k} \lambda^{i+j}}{(r-1) !(s-r-1) !} \sum_{u=0}^{r-1} \sum_{v=0}^{s-r-1} \sum_{p=0}^{\frac{j}{\alpha}} \sum_{q=0}^{\frac{i}{\alpha}} \frac{(-1)^{r-1-u+v+\frac{(i+j)}{\alpha}-p-q}}{k^{\beta p+s-r-v}} \times\left(\begin{array}{c}
r-1 \\
u
\end{array}\right)\left(\begin{array}{c}
s-r-1 \\
v
\end{array}\right)\left(\begin{array}{c}
\frac{j}{\alpha} \\
p
\end{array}\right)\left(\begin{array}{c}
\frac{i}{\alpha} \\
q
\end{array}\right) \times \\
& \int_{0}^{\infty} w^{\beta q+u+v} \Gamma(\beta p+s-r-v, k w) d w
\end{aligned}
$$

where $\mathrm{w}=\left[1+\left(\frac{x}{\lambda}\right)^{\alpha}\right]^{\frac{1}{\beta}}$. The result follows by using equation (6.455.1) in Gradshteyn and Ryzhik(2007) to calculate the integral in (26).

The proof is complete. 


\section{Special Cases.}

1) For $m=O$ and $k=1$ in (23), we get the explicit expression for product moments of ordinary order statistics

$$
\begin{aligned}
& \mu_{r, s ; n}^{(i, j)}=\lambda^{i+j} C_{r, s: n} \sum_{u=0}^{r-1} \sum_{v=0}^{s-r-1} \sum_{p=0}^{j / \alpha} \sum_{q=0}^{i / \alpha}(-1)^{u+v+\frac{i+j}{\alpha}-p-q}\left(\begin{array}{c}
r-1 \\
u
\end{array}\right) \times \\
& \left(\begin{array}{c}
s-r-1 \\
v
\end{array}\right)\left(\begin{array}{c}
j / \alpha \\
p
\end{array}\right)\left(\begin{array}{c}
i / \alpha \\
q
\end{array}\right) \frac{e^{n-r+u+1}}{(\beta q+1)(n-r+u+1)^{2+\beta(p+q)}} \times 2 F_{1}\left(1,2+\beta(p+q) ; 2+\beta q ; \frac{(u+s-r-v)}{(n-r+u+1)}\right),
\end{aligned}
$$

which is the result obtained by Kumar and Sanku (2017).

In particular, the covariance of order statistics is

$$
\begin{aligned}
& \mu_{r, s ; n}^{(1,1)}=\lambda^{2} C_{r, s: n} \sum_{u=0}^{r-1} \sum_{v=0}^{s-r-1} \sum_{p=0}^{1 / \alpha} \sum_{q=0}^{1 / \alpha}(-1)^{u+v+\frac{2}{\alpha}-p-q}\left(\begin{array}{c}
r-1 \\
u
\end{array}\right) \times \\
& \left(\begin{array}{c}
s-r-1 \\
v
\end{array}\right)\left(\begin{array}{c}
1 / \alpha \\
p
\end{array}\right)\left(\begin{array}{c}
1 / \alpha \\
q
\end{array}\right) \frac{e^{n-r+u+1}}{(\beta q+1)(n-r+u+1)^{2+\beta(p+q)}} \times 2 F_{1}\left(1,2+\beta(p+q) ; 2+\beta q ; \frac{(u+s-r-v)}{(n-r+u+1)}\right) .
\end{aligned}
$$

2) For $\mathrm{k}=1$ in (24), we get explicit expression for record values

$$
\begin{aligned}
\mu_{U_{r, s}}^{(i, j)}=\frac{\lambda^{i+j} e}{(r-1) !(s-r-1)} \sum_{u=0}^{r-1} \sum_{v=0}^{s-r-1} \sum_{p=0}^{j / \alpha} \sum_{q=0}^{i / \alpha}(-1)^{\frac{i+j}{\alpha}+r+v-u-p-q-1} \\
\quad \times\left(\begin{array}{c}
r-1 \\
u
\end{array}\right)\left(\begin{array}{c}
s-r-1 \\
v
\end{array}\right)\left(\begin{array}{c}
j / \alpha \\
p
\end{array}\right)\left(\begin{array}{c}
i / \alpha \\
q
\end{array}\right) \frac{\Gamma(\beta(p+q)+s-r+u+1)}{(\beta q+u+v+1)} \\
\times 2 F_{1}(1, \beta(p+q)+s-r+u+1 ; \beta q+u+v+2 ; 1) .
\end{aligned}
$$

Theorem 5. For the PGW distribution given in (1) and for $1 \leq r<s \leq n$ and $i=0,1$, $2, \ldots$ and $j$ a negative integer,

$$
\begin{aligned}
\mu_{r, s ; n, m, k}^{(i, j)}=\frac{\lambda^{i+j} C_{s-1}}{(r-1) !(s-r-1) !(m+1)^{s-1}} \sum_{u=0}^{r-1} \sum_{v=0}^{s-r-1} \sum_{p=0}^{\infty} \sum_{q=0}^{i / \alpha}(-1)^{u+v+\frac{i+j}{\alpha}-p-q} \\
\quad \times\left(\begin{array}{c}
r-1 \\
u
\end{array}\right)\left(\begin{array}{c}
s-r-1 \\
v
\end{array}\right)\left(\begin{array}{c}
j / \alpha \\
p
\end{array}\right)\left(\begin{array}{c}
i / \alpha \\
q
\end{array}\right) \frac{e^{\gamma_{r-u}}}{(\beta q+1) \gamma_{r-u}^{2+\beta(p+q)}} \\
\times 2 F_{1}\left(1,2+\beta(p+q) ; 2+\beta q ; \frac{(u+s-r-v)(m+1)}{\gamma_{r-u}}\right), m \neq 1
\end{aligned}
$$

and for $\mathrm{m}=-1$ 


$$
\begin{aligned}
\mu_{r, s ; n,-1, k}^{(i, j)}=\frac{\lambda^{i+j} e^{k} k^{s}}{(r-1) !(s-r-1) !} \sum_{u=0}^{r-1} \sum_{v=0}^{s-r-1} \sum_{p=0}^{\infty} \sum_{q=0}^{i / \alpha}(-1)^{\frac{i+j}{\alpha}+r+v-u-p-q-1} \\
\quad \times\left(\begin{array}{c}
r-1 \\
u
\end{array}\right)\left(\begin{array}{c}
s-r-1 \\
v
\end{array}\right)\left(\begin{array}{c}
j / \alpha \\
p
\end{array}\right)\left(\begin{array}{c}
i / \alpha \\
q
\end{array}\right) \frac{\Gamma(\beta(p+q)+s-r+u+1)}{(\beta q+u+v+1)} \\
\times 2 F_{1}(1, \beta(p+q)+s-r+u+1 ; \beta q+u+v+2 ; 1),
\end{aligned}
$$

where $2 F_{1}(a, b, c ; x)$ denotes the Gauss hypergeometric function defined by

$$
2 F_{1}(a, b, c ; x)=\sum_{k=0}^{\infty} \frac{(a)_{k}(b)_{k}}{(c)_{k}} \frac{x^{k}}{k !},
$$

where $(e)_{k}=e(e+1) \ldots(e+k-1)$ denotes the ascending fractorial.

Proof. Similar to the proof of Theorem 4.

Theorem 6. For the PGW distribution given in (1) and for $1 \leq r<s \leq n$ and $j=0,1$, $2, \ldots$ and $i$ a negative integer,

$$
\begin{aligned}
\mu_{r, s ; n, m, k}^{(i, j)}=\frac{\lambda^{i+j} C_{s-1}}{(r-1) !(s-r-1) !(m+1)^{s-2}} \sum_{u=0}^{r-1} \sum_{v=0}^{s-r-1} \sum_{p=0}^{\frac{j}{\alpha}} \sum_{q=0}^{\infty}(-1)^{u+v+\frac{i+j}{\alpha}-p-q} \\
\times\left(\begin{array}{c}
r-1 \\
u
\end{array}\right)\left(\begin{array}{c}
s-r-1 \\
v
\end{array}\right)\left(\begin{array}{c}
\frac{j}{\alpha} \\
p
\end{array}\right)\left(\begin{array}{c}
\frac{i}{\alpha} \\
q
\end{array}\right) \frac{e^{\gamma_{r-u}}}{(\beta q+1) \gamma_{r-u}^{2+\beta(p+q)}} \\
\times 2 F_{1}\left(1,2 \beta(p+q) ; 2+\beta q ; \frac{(u+s-r-v)(m+1)}{\gamma_{r-u}}\right), m \neq-1
\end{aligned}
$$

and for $m=-1$

$$
\begin{aligned}
\mu_{r, s ; n,-1, k}^{(i, j)}=\frac{\lambda^{i+j} e^{k} k^{s}}{(r-1) !(s-r-1) !} \sum_{u=0}^{r-1} \sum_{v=0}^{s-r-1} \sum_{p=0}^{j / \alpha} \sum_{q=0}^{\infty}(-1)^{\frac{i+j}{\alpha}+r+v-u-p-q-1} \\
\quad \times\left(\begin{array}{c}
r-1 \\
u
\end{array}\right)\left(\begin{array}{c}
s-r-1 \\
v
\end{array}\right)\left(\begin{array}{c}
\frac{j}{\alpha} \\
p
\end{array}\right)\left(\begin{array}{c}
\frac{i}{\alpha} \\
q
\end{array}\right) \frac{\Gamma(\beta(p+q)+s-r+u+1)}{(\beta q+u+v 1)} \\
\quad \times 2 F_{1}(1, \beta(p+q)+s-r+u+1 ; \beta q+u+v+2 ; 1)
\end{aligned}
$$

Proof. Similar to the proof of Theorem 4. 
Theorem 7.For the PGW distribution given in (1) and for $1 \leq r<s \leq n$ and both $i$ and $j$ negative integers,

$$
\begin{aligned}
\mu_{r, s ; n, m, k}^{(i, j)}=\frac{\lambda^{i+j} C_{s-1}}{(r-1) !(s-r-1) !(m+1)^{s-2}} \sum_{u=0}^{r-1} \sum_{v=0}^{s-r-1} \sum_{p=0}^{\infty} \sum_{q=0}^{\infty}(-1)^{u+v+\frac{i+j}{\alpha}-p-q} \\
\quad \times\left(\begin{array}{c}
r-1 \\
u
\end{array}\right)\left(\begin{array}{c}
s-r-1 \\
v
\end{array}\right)\left(\begin{array}{c}
j / \alpha \\
p
\end{array}\right)\left(\begin{array}{c}
i / \alpha \\
q
\end{array}\right) \frac{e^{\gamma_{r-u}}}{(\beta q+1) \gamma_{r-u}^{2+\beta(p+q)}} \\
\quad \times 2 F_{1}\left(1,2+\beta(p+q) ; 2+\beta q ; \frac{(u+s-r-v)(m+1)}{\gamma_{r-u}}\right), m \neq-1
\end{aligned}
$$

and for $m=-1$

$$
\begin{aligned}
\mu_{r, s ; n,-1, k}^{(i, j)}=\frac{\lambda^{i+j} e^{k} k^{s}}{(r-1) !(s-r-1) !} \sum_{u=0}^{r-1} \sum_{v=0}^{s-r-1} \sum_{p=0}^{\infty} \sum_{q=0}^{\infty}(-1)^{u+v+\frac{i+j}{\alpha}+r+v-u-p-q-1} \\
\quad \times\left(\begin{array}{c}
r-1 \\
u
\end{array}\right)\left(\begin{array}{c}
s-r-1 \\
v
\end{array}\right)\left(\begin{array}{c}
j / \alpha \\
p
\end{array}\right)\left(\begin{array}{c}
i / \alpha \\
q
\end{array}\right) \frac{\Gamma(\beta(p+q)+s-r+u+1)}{(\beta q+u+v+1)} \\
\times 2 F_{1}(1, \beta(p+q)+s-r+u+1 ; \beta q+u+v+2 ; 1),
\end{aligned}
$$

Proof. Similar to the proof of Theorem 4.

Theorem 8 establishes a recurrence relation for $\mu(i, j)$. This result holds for positive as well as negative values of $i$ and $j$.

Theorem 8. For the PGW distribution given in (1) and for $1 \leq r<s \leq n$,

$$
\mu_{r, s ; n, m, k}^{(i, j)}=\frac{\alpha}{\beta} \sum_{u=0}^{\infty}\left(\begin{array}{c}
\frac{1}{\beta}-1 \\
u
\end{array}\right) \frac{\lambda^{-\alpha(u+1)}}{j+\alpha(u+1)} \times\left[\gamma_{s} \mu_{r, s, n, m, k}^{(i, j+\alpha(u+1)}-(s-r-1) \mu_{r, s-1, n, m, k}^{(i, j+\alpha(u+1)}\right]
$$

Proof. For $1 \leq r \leq n$, we have from (4) and (7)

$$
\mu_{r, s, n, m, k}^{(i, j)}=\frac{c_{s-1}}{(r-1) !(s-r-1) !} \int_{0}^{\infty} x^{i}[\bar{F}(x)]^{m} g_{m}^{r-1}(F(x)) G(x) f(x) d x
$$

where

$$
\begin{aligned}
\mathrm{G}(\mathrm{x})=\int_{x}^{\infty} y^{j}[ & \left.h_{m}(F(y))-h_{m}(F(x))\right]^{s-r-1}[\bar{F}(y)]^{\gamma_{s}-1} f(y) d y \\
= & \frac{\alpha}{\beta} \sum_{u=0}^{\infty}\left(\begin{array}{c}
\frac{1}{\beta}-1 \\
u
\end{array}\right) \frac{1}{\lambda^{\alpha(u+1)}} \int_{x}^{\infty} y^{j+\alpha(u+1)-1}[\bar{F}(y)]^{\gamma_{s}}\left[h_{m}(F(y))-h_{m}(F(x))\right]^{s-r-1} d y
\end{aligned}
$$

By integrating by parts with respect to $y$, we obtain 


$$
\begin{aligned}
\mathrm{G}(\mathrm{x})=\frac{\alpha}{\beta} \sum_{u=0}^{\infty}\left(\begin{array}{c}
\frac{1}{\beta}-1 \\
u
\end{array}\right) \frac{\lambda^{-\alpha(u+1)}}{j+\alpha(u+1)}\left[\gamma _ { s } \int _ { x } ^ { \infty } y ^ { j + \alpha ( u + 1 ) } [ \overline { F } ( y ) ] ^ { \gamma _ { s } - 1 } \left[h_{m}(F(y))\right.\right. \\
\left.\quad-h_{m}(F(x))\right]^{s-r-1} f(y) d y-(s-r \\
\left.\quad-1) \int_{x}^{\infty} y^{j+\alpha(u+1)}[\bar{F}(y)]^{\gamma_{s}+m}\left[h_{m}(F(y))-h_{m}(F(x))\right]^{s-r-2} f(y) d y\right] .
\end{aligned}
$$

The result follows by combining (36) and (37).

In particular, upon setting $\mathrm{s}=\mathrm{r}+1$ in Theorem 8 , we deduce the following result.

Corollary2. For the distribution given in (1) and $1 \leq \mathrm{r}<\mathrm{n}, \mathrm{n} \geq 1, \mathrm{k}=1,2, \ldots$,

$$
\mu_{r, r+1, n, m, k}^{(i, j)}=\frac{\alpha}{\beta} \sum_{u=0}^{\infty}\left(\begin{array}{c}
\frac{1}{\beta}-1 \\
u
\end{array}\right) \frac{\lambda^{-\alpha(u+1)} \gamma_{r+1}}{j+\alpha(u+1)} \mu_{r, r+1, n, m, k}^{(i, j+\alpha(u+1))} .
$$

\section{Special Cases}

1) For $\mathrm{m}=0, \mathrm{k}=1$ in (35), we get the recurrence relations for ordinary order statistics

$$
\mu_{r, s: n}^{(i, j)}=\frac{\alpha}{\beta} \sum_{u=0}^{\infty}\left(\begin{array}{c}
\frac{1}{\beta}-1 \\
u
\end{array}\right) \frac{\lambda^{-\alpha(u+1)}}{j+\alpha(u+1)} \times\left[(n-s+1) \mu_{r, s-1: n}^{(i, j+\alpha(u+1))}\right] .
$$

2) For $\mathrm{m}=-1, \mathrm{k} \geq 1,(35)$, we get the recurrence relation for record values

$$
\mu_{U(r, s): n}^{(i, j)}=\frac{\alpha}{\beta} \sum_{u=0}^{\infty}\left(\begin{array}{c}
\frac{1}{\beta}-1 \\
u
\end{array}\right) \frac{\lambda^{-\alpha(u+1)}}{j+\alpha(u+1)} \times\left[k \mu_{U(r, s): n}^{(i, j+\alpha(u+1))}\right] .
$$

\subsection{Relations for conditional moments}

Let $\mathrm{X}(\mathrm{r}, \mathrm{n}, \mathrm{m}, \mathrm{k}), \mathrm{r}=1,2, \ldots, \mathrm{n}$ be gos, then from a continuous population with $\mathrm{cdf} F(\mathrm{x})$ and $\operatorname{pdf} \mathrm{f}$ (x), then the conditional pdf of $X(s, n, m, k)$ given $X(r, n, m, k)=x, 1 \leq r<s \leq n$, in view of (6) and (7), is

$$
f_{s \mid r}(y \mid x)=\frac{C_{s-1}}{(s-r-1) ! C_{r-1}} \frac{\left[h_{m}(F(y))-h_{m}(F(x))\right]^{s-r-1}[\bar{F}(y)]^{\gamma_{s}-1}}{[\bar{F}(x)]^{\gamma r+1}} f(y), y>x,
$$

and the conditional pdf of $\mathrm{X}(\mathrm{r}, \mathrm{n}, \mathrm{m}, \mathrm{k})$ given $\mathrm{X}(\mathrm{s}, \mathrm{n}, \mathrm{m}, \mathrm{k})=\mathrm{y}, 1 \leq \mathrm{r}<\mathrm{s} \leq \mathrm{n}$

$$
\begin{array}{r}
f_{r \mid s}(x \mid y)=\frac{(s-1) !(m+1)[\bar{F}(x)]\left[1-(\bar{F}(x))^{m+1}\right]^{r-1}}{(r-1) !(s-r-1) !} \\
\times \frac{\left[(\bar{F}(x))^{m+1}-(\bar{F}(y))^{m+1}\right]}{\left[1-(\bar{F}(y))^{m+1}\right]^{s-1}} f(x), y>x .
\end{array}
$$

We shall first establish conditional moments of GOS, $X(s, n, m, k)$ given $X(r, n, m, k)=x$ i.e. $\mathrm{E}\left[X^{(j)}(\mathrm{s}, \mathrm{n}, \mathrm{m}, \mathrm{k}) \mid \mathrm{X}(\mathrm{r}, \mathrm{n}, \mathrm{m}, \mathrm{k})=\mathrm{x}\right]=\mu_{s, n, m, k \mid r, n, m, k}^{(j)}$ and $X(r, n, m, k)$ given $X(s, n, m, k)=y$ i.e $\mathrm{E}\left[X^{(j)}(\mathrm{s}, \mathrm{n}, \mathrm{m}, \mathrm{k}) \mid \mathrm{X}(\mathrm{r}, \mathrm{n}, \mathrm{m}, \mathrm{k})=\mathrm{y}\right]=\mu_{s, n, m, k \mid r, n, m, k}^{(j)}$. Theorem 9 gives the conditional moments of 
GOS, $X(s, n, m, k)$ given $X(r, n, m, k)=x$ for $1 \leq r<s \leq n$ and $j=0,1,2, \ldots$ Theorem 10 gives the conditional moments of GOS, $X(r, n, m, k)$ given $X(s, n, m, k)=y$ for $1 \leq r<s \leq n$ and $j=0,1,2, \ldots$

Theorem 9. For the distribution given in (1) and $1 \leq r<s \leq n, n \geq 1, k=1,2, \ldots$ and $i, j=0,1$, $2, \ldots$

$$
\begin{aligned}
\mu_{s, n, m, k \mid r, n, m, k}^{(j)}= & \frac{\lambda^{j} C_{s-1}}{(s-r-1) ! C_{\mathrm{r}-1}(m+1)^{s-r-1}} \\
& \times \sum_{u=0}^{s-r-1} \sum_{p=0}^{j / \alpha}(-1)^{u+\frac{j}{\alpha}-p}\left(\begin{array}{c}
s-r-1 \\
u
\end{array}\right)\left(\begin{array}{c}
j / \alpha \\
p
\end{array}\right) \\
& \times \frac{e^{\gamma_{s-u}} \Gamma\left(\beta p+1, \gamma_{s-u}\left[1+\left(\frac{x}{\lambda}\right)^{\alpha}\right]^{\frac{1}{\beta}}\right)}{\gamma_{s-u}^{\beta p+1} e^{\left[1+\left(\frac{x}{\lambda}\right)^{\alpha}\right]^{\frac{1}{\beta}} \gamma_{s}}}
\end{aligned}
$$

Proof. Similar to the proof of Theorem 4.

Theorem 10. For the distribution given in (1) and $1 \leq r \quad<s \leq n, \quad n \geq 1, k=1,2, \ldots$ andi, $j=0,1$, $2, \ldots$

$$
\begin{aligned}
\mu_{r, n, m, k \mid s, n, m, k}^{(j)}= & \frac{\lambda^{j}(s-1) !(m+1) e^{\left\{1-\left[1+\left(\frac{y}{\lambda}\right)^{\alpha}\right]^{\frac{1}{\beta}}\right\}(m+1) u}}{(r-1) !(s-r-1) !\left[1-e^{\left\{1-\left[1+\left(\frac{y}{\lambda}\right)^{\alpha}\right]^{\frac{1}{\beta}}\right\}(m+1)}\right]^{s-1}} \\
& \times \sum_{u=0}^{s-r-1} \sum_{v=0}^{r-1} \sum_{p=0}^{j / \alpha}(-1)^{u+v+\frac{j}{\alpha}-p}\left(\begin{array}{c}
s-r-1 \\
u
\end{array}\right)\left(\begin{array}{c}
r-1 \\
v
\end{array}\right) \times\left(\begin{array}{c}
\frac{j}{\alpha} \\
p
\end{array}\right) \frac{e^{\varepsilon}}{\varepsilon^{\beta p+1}} \Gamma(\beta p \\
& \left.+1, \varepsilon\left[1+\left(\frac{x}{\lambda}\right)^{\alpha}\right]^{\frac{1}{\beta}}\right)
\end{aligned}
$$

where $\xi=(s-r-u+v)(m+1)$ and $\Gamma(\mathrm{a}, \mathrm{x})$ denotes the incomplete gamma function defined by $\Gamma(\mathrm{a}, \mathrm{x})=\int_{x}^{\infty} t^{a-1} e^{-t} d t$

Proof: Similar to the proof of Theorem1.

Remark 1: For $\mathrm{k}=1, \mathrm{~m}=0$ and $\mathrm{k}=1, \mathrm{~m}=-1$ in (43) and (44), we obtain the conditional momemts of order statistics and record values, respectively.

\section{Characterization}

In this section, we characterize the PGW distribution based on conditional moments of order statistics. Let $\mathrm{L}(\mathrm{a}, \mathrm{b})$ denote the space of all integrable functions on $(\mathrm{a}, \mathrm{b})$. A sequence $\left(h_{n}\right) \subset L(a, b)$ is called complete on $\mathrm{L}(\mathrm{a}, \mathrm{b})$ if for all functions $\mathrm{g} \in \mathrm{L}(\mathrm{a}, \mathrm{b})$ the condition

$$
\int_{a}^{b} g(x) f_{n}(x) d x=0, n \in \mathrm{N}
$$

implies $g(x)=0$ a.e. on (a, b). We start with the following result of Lin (1986). 
Proposition 1. Let $n_{0}$ be any fixed non-negative integer, $-\infty \leq \mathrm{a}<\mathrm{b} \leq \infty$ and $\mathrm{g}(\mathrm{x}) \geq 0$ a.n absolutely continuou function with $g^{\prime}(x) \neq 0$ a.e on $(a, b)$. Then the sequence of functions $\left\{(\mathrm{g}(\mathrm{x}))^{n} e^{-g(x)}, \mathrm{n} \geq n_{0}\right\}$ is complete in $\mathrm{L}(\mathrm{a}, \mathrm{b})$ if and only if $\mathrm{g}(\mathrm{x})$ is strictly monotone on $(\mathrm{a}, \mathrm{b})$.

Using the above proposition, we obtain a stronger version of Theorem 3.

Theorem 11. Let $X$ be a non-negative random variable having an absolutely continuous cdf $F$ $(x)$ with $F(O)=O$ and $0<F(x)<1$ for all $\mathrm{x}>0$. Then

$$
\mu_{r, n, m, k}^{(j)}=\frac{\alpha}{\beta} \sum_{u=0}^{\infty}\left(\begin{array}{c}
\frac{1}{\beta}-1 \\
u
\end{array}\right) \frac{\lambda^{-\alpha(u+1)}}{j+\alpha(u+1)} \times\left[\gamma_{r} \mu_{r, n, m, k}^{(j+\alpha(u+1))}-(r-1) \mu_{r-1, n, m, k}^{(j+\alpha(u+1))}\right] .
$$

if and only if

$$
\mathrm{F}(\mathrm{x})=1-e^{1-\left[1+\left(\frac{x}{\lambda}\right)^{\alpha}\right]^{\frac{1}{\beta}}} ; x>0, \alpha, \beta, \lambda>0
$$

Proof. The necessary part follows immediately from equation (17). On the other hand if the recurrence relation in equation (45) is satisfied, then on using equations (4), we have

$$
\begin{aligned}
\frac{C_{r-1}}{(r-1) !} \int_{0}^{\infty} x^{j}[\bar{F}(x)]^{\gamma_{r}-1} g_{m}^{r-1}(F(x)) f(x) d x \\
=\frac{C_{r-1}}{(r-1) !} \frac{\alpha}{\beta}\left(\begin{array}{c}
\frac{1}{\beta}-1 \\
u
\end{array}\right) \frac{\lambda^{-\alpha(u+1)}}{\alpha(u+1+j)} \\
\quad \times\left\{\gamma_{r} \int_{0}^{\infty} x^{j+\alpha(u+1)}[\bar{F}(x)]^{\gamma_{r}-1} g_{m}^{r-1}(F(x)) d x\right. \\
\left.-(r-1) \int_{0}^{\infty} x^{j+\alpha(u+1)}[\bar{F}(x)]^{\gamma_{r-1}-1} g_{m}^{r-1}(F(x)) f(x) d x\right\} .
\end{aligned}
$$

Integrating by parts the last integral on the right hand side of equation (46), we get

$$
\begin{aligned}
\frac{C_{r-1}}{(r-1) !} \int_{0}^{\infty} x^{j}[\bar{F}(x)]^{\gamma_{r}-1} g_{m}^{r-1}(F(x)) f(x) d x \\
=\frac{C_{r-1}}{(r-1) !} \frac{\alpha}{\beta}\left(\begin{array}{c}
\frac{1}{\beta}-1 \\
u
\end{array}\right) \frac{\lambda^{-\alpha(u+1)}}{\alpha(u+1+j)} \times\left\{\gamma_{r} \int_{0}^{\infty} x^{j+\alpha(u+1)}[\bar{F}(x)]^{\gamma_{r}-1} g_{m}^{r-1}(F(x)) d x\right. \\
-\gamma_{r} \int_{0}^{\infty} x^{j+\alpha(u+1)}[\bar{F}(x)]^{\gamma_{r}-1} g_{m}^{r-1}(F(x)) f(x) d x-j+\alpha(u \\
+1) \int_{0}^{\infty} x^{j+\alpha(u+1)}[\bar{F}(x)]^{\gamma_{r}} g_{m}^{r-1}(F(x)) d x .
\end{aligned}
$$

which reduces to

$$
\left.\frac{C_{r-1}}{(r-1) !} \int_{0}^{\infty} x^{j}[\bar{F}(x)]^{\gamma_{r}-1} g_{m}^{r-1}(F(x))\{f(x)\}-\frac{\alpha}{\beta} \sum_{u=0}^{\infty}\left(\begin{array}{c}
\frac{1}{\beta}-1 \\
u
\end{array}\right) \frac{x^{\alpha(u+1)-1}}{\lambda^{\alpha(u+1)}} \bar{F}(x)\right\} d x=0
$$


It now follows from Proposition 1

$$
\mathrm{f}(\mathrm{x})=\frac{\alpha}{\beta} \sum_{u=0}^{\infty}\left(\begin{array}{c}
\frac{1}{\beta}-1 \\
u
\end{array}\right) \frac{x^{\alpha(u+1)-1}}{\lambda^{\alpha(u+1)}} \bar{F}(x)
$$

or

$$
\frac{f(x)}{\bar{F}(x)}=\frac{\alpha}{\beta} \sum_{u=0}^{\infty}\left(\begin{array}{c}
\frac{1}{\beta}-1 \\
u
\end{array}\right) \frac{x^{\alpha(u+1)-1}}{\lambda^{\alpha(u+1)}}
$$

which proves that

$$
\mathrm{F}(\mathrm{x})=1-e^{1-\left[1+\left(\frac{x}{\lambda}\right)^{\alpha}\right]^{\frac{1}{\beta}}} ; x>0, \alpha, \beta, \lambda>0
$$

\section{$5 \quad$ Numerical Computation}

The recurrence relations obtained in the preceding sections allow us to evaluate the means and variances of all order statistics and record values for all sample sizes in a simple recursive manner. In Figure 3, we have presented the means of all order statistics for sample size $n=1(1) 5$ and $\alpha=$ $0.5(0.5) 3.5, \beta=0.5$ and $\lambda=0.5$ and we observe that the mean of order statistics decreasing as $\alpha$ increasing. The variances are presentation in Figure 4 of all order statistics for different values of $r$ and $\mathrm{n}$ for $\alpha=0.5(0.5) 3.5, \beta=0.5$ and $\lambda=0.5$ and we observe that as $\alpha$ increases, variances of order statistics decreases. From Figure 5-6, similar conclusion can be drawn for mean and variances of record values. Tabular values of means and variances of order statistics and record values are presented in Appendix.
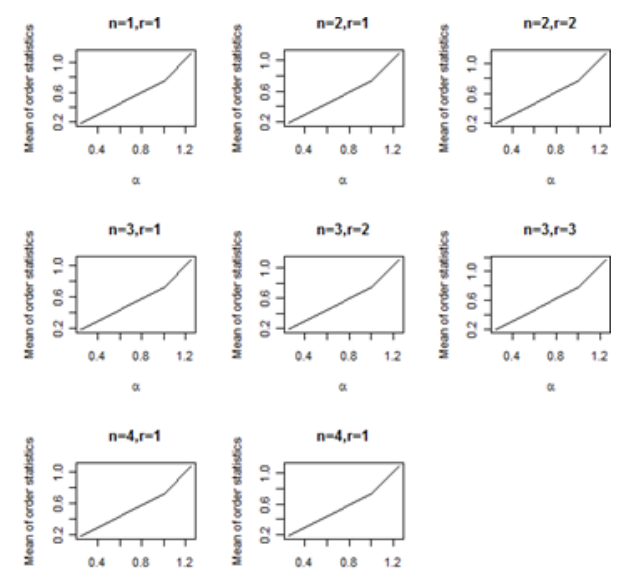
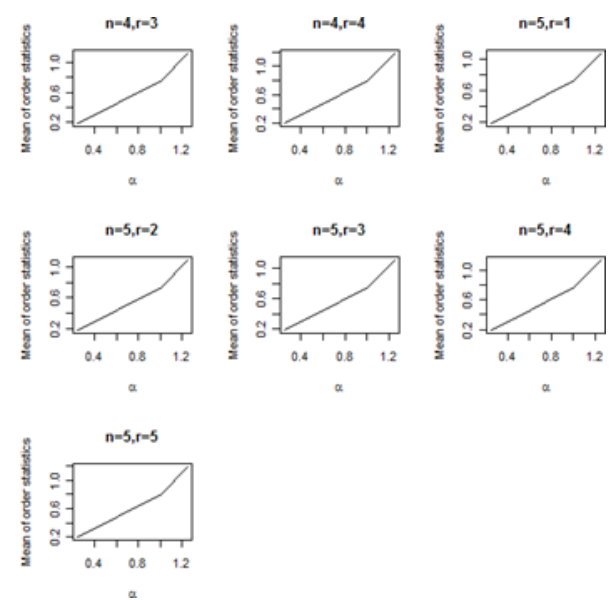

Figure 3: Mean of order statistics for $\alpha=0.5,1.0,1.5,2.0,2.5,3.0,3.5$ 

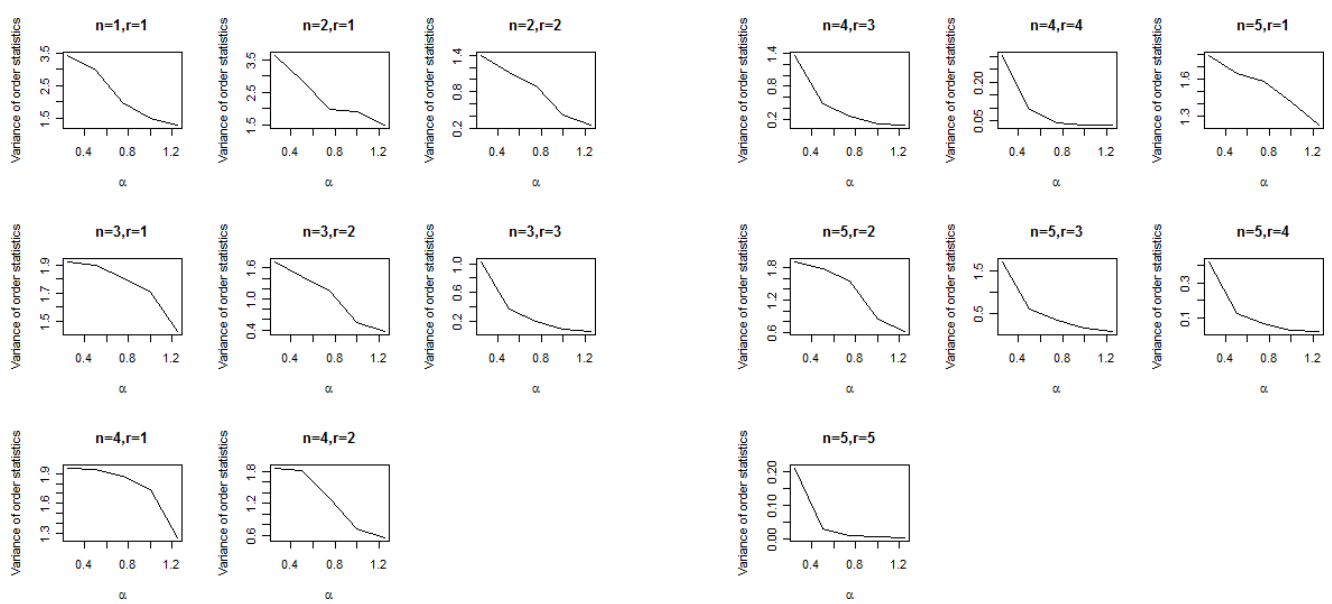

Figure 4: Variance of order statistics for $\alpha=0.5,1.0,1.5,2.0,2.5,3.0,3.5$.
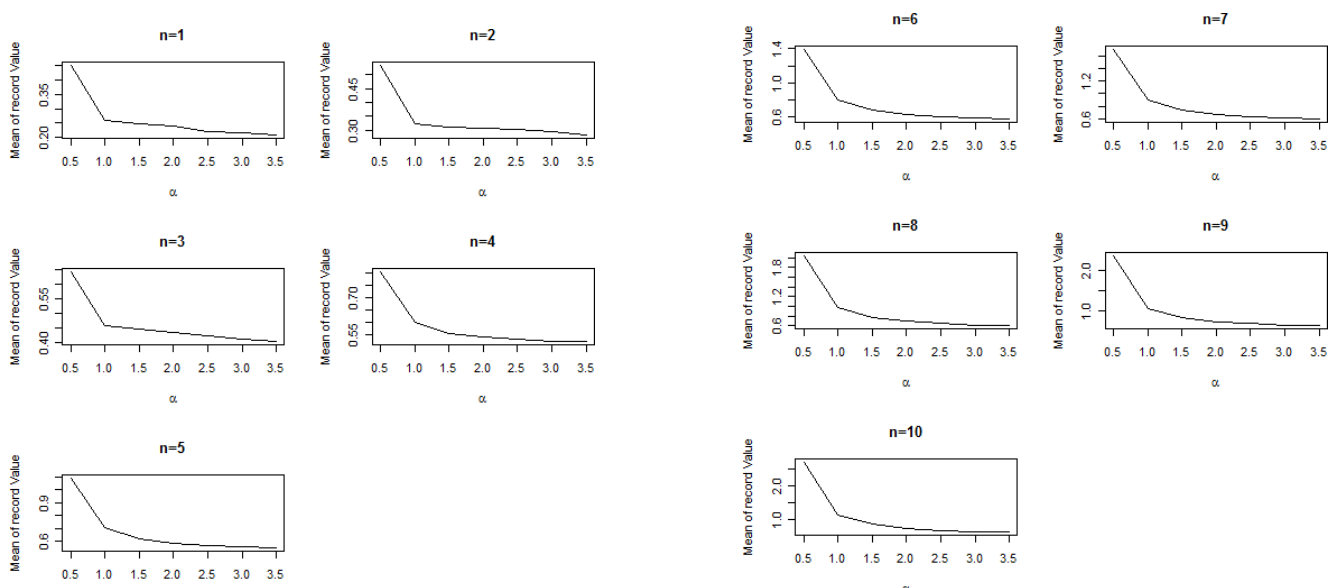

Figure 5: Mean of record values for $\alpha=0.5,1.0,1.5,2.0,2.5,3.0,3.5$.
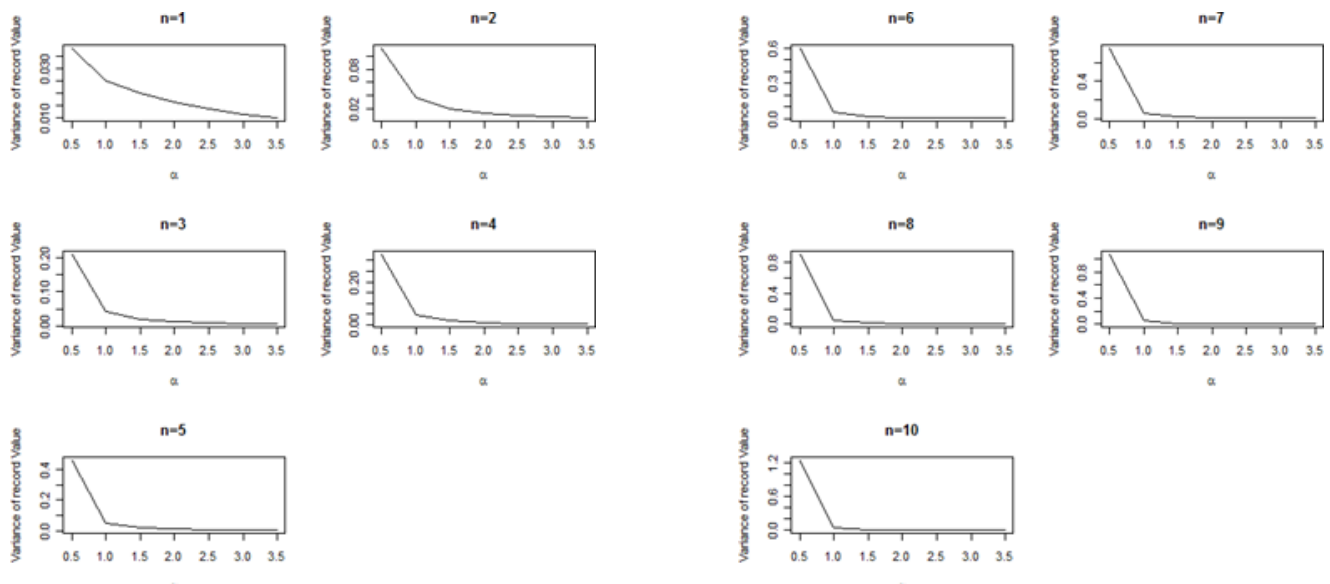

Figure 6: Variance of record values for $\alpha=0.5,1.0,1.5,2.0,2.5,3.0,3.5$. 


\section{Discussion}

In this paper, recurrence relations for single and product moments of generalized order statistics from PGW distribution have been derived. The expressions of conditional moments of general- ized order statistics from PGW distribution have also been derived. One characterization of the distributions is shown. The numerical computations of moments are shown for order statistics as well as record values and we see that the moments of order statistics and record values of the distribution are well behaved. This will encourage the study of the other properties of generalized order statistics for future scope of research.

\section{Acknowledgements}

The authors would like to thank the reviewrs and editor for their comments which helped improve the paper.

\section{References}

[1] Alloyarova, R., Nikulin, M., Pya, N., Voinov, V. (2007). The power-generalized Weibull probability distribution and its use in survival analysis. Communications in Dependability and Quality Management, 10, 5-15.

[2] Arnold, B. C., Balakrishnan, N. and Nagaraja, H. N. (1992). A First Course in Order Statistics. John Wiley, New York.

[3] Bagdonavi`cius, V. and Nikulin, M., (2002). Accelerated Life Models. Chapman and Hall/CRC, Boca Raton.

[4] Bagdonavi cius, Clerjaud, L., Nikulin, M.,(2006). Power generalized Weibull in accelerated life testing. Preprint 0602, Statistique Mathematique et ses Applications, I.F.R. 99, Université Victor Segalen Bordeaux 2, p. 28.

[5] Balakrishnan, N., Zhu, X. and Al-Zaharani, B., (2015). Recursive computation of the single and product moments of order statistics for the complementary exponential-geometric distribution. Journal of Statistical Computation and Simulation, 85 , 2187-2201. 
[6] Bebbington, M., Lai, C.D. and Zitikis, R. (2007). A flexible weibull extension. Reliability Engineering and System Safety, 92, 719-726.

[7] Cordeiro, G.M., Edwin, M.M. Ortega and Nadarajah, S., (2010). The Kumaraswamy Weibull distribution with application to failure data. J. Franklin Inst., 347 , 1399-1429.

[8] Efron, B., (1988). Logistic regression, survival analysis, and the kaplan-meier curve. Journal of the American Statistical Association, 83, 414-425.

[9] Ghitany M.E., Al-Hussaini E.K. and Al-Jarallah R.A., (2005). Marshall-Olkin extended Weibull distribution and its application to censored data. J. Appl. Stat. 32, 1025-1034.

[10] Gradshteyn, I. S. and Ryzhik, I. M., (2000). Table of Integrals, Series, and Products, sixth edition. Academic Press, San Diego.

[11] Kamps, U. (1995): A concept of generalized order statistics. B.G. Teubner Stuttgart.

[12] Kumar, D., (2013). Relations for marginal and joint moment generating functions of Marshall Olkin extended logistic distribution based on lower generalized order statistics and characterization. American Journal of Mathematical and Management Sciences, 32, 19-39.

[13] Kumar, D., (2014). On moment generating function of generalized order statistics from extended type II generalized logistic distribution. Journal of Statistical Theory and Application, $13,135-150$.

[14] Kumar, D. (2015): Ratio and inverse moments of generalized order statistics of Marshall Olkin extended Bur type XII Distribution. Journal of Data Science, 14, 53-66. 
[15] Kumar, D., (2015). Exact moments of generalized order statistics from type II exponentiated log-logistic distribution. Hacettepe Journal of Mathematics and Statistics, $44,715-733$.

[16] Kumar, D., Dey, S., and Nadarajah, S., (2017 ). Extended exponential distribution based on order statistics, Communications in Statistics-Theory and Methods, 46, 9166-9184.

[17] Kumar, D. and Dey, S. (2017). Power generalized Weibull distribution based on order statistics, Journal of Statistical Research, 51, 61-78.

[18] Kumar, D. and Dey, S. (2017). Relations for moments of generalized order statistics from extended exponential distribution, American Journal of Mathematical and Management Sciences, 36(4), 378-400.

[19] Kumar, D., (2017): The Burr type XII distribution with some statistical properties, Journal of Data Science, 15, 509-533.

[20] Lin, G. D., (1988). Characterizations of distributions via relationships between two moments of order statistics. Journal of Statistical Planning and Inference, 19, 73-80.

[21] Mudholkar, G.S. and Srivastava, D.K., (1993). Exponentiated Weibull family for analyzing bathtub failure-real data, IEEE Transaction Reliability, 42, 299-302.

[22] Nikulin, N and Haghighi, F., (2009). On the power generalized Weibull family: model for cancer censored data, Metron, LXVII, 75-86.

[23] Ristić, M. M. and Balakrishnan, N. (2012). The gamma-exponentiated exponential distribution. J. Statist. Comput., Simul., 82 , 1191-1206. 
[24] Silva, G.O., Edwin, M. M. Ortega and Cordeiro, G. M., (2010). The beta modified Weibull distribution. Lifetime Data Anal., 16, 409-430.

[25] Voinov, V, Pya, N, Shapakov, N, Voinov, Y. (2013). Goodness-of-fit tests for the powergeneralized weibull probability distribution. Communications in Statistics-Simulation and Computation, 42 , 1003-1012.

[26] Wahed, Abdus S., Luong, The Minh and Jeong, Jong-Hyeon. (2009). A new generalization of Weibull distribution with application to a breast cancer data set. Stat Med. 28, 2077-2094. 